\title{
Necessary and Sufficient Dissipativity-Based Conditions for Feedback Stabilization
}

This paper was downloaded from TechRxiv (https://www.techrxiv.org).

LICENSE

CC BY-NC-SA 4.0

SUBMISSION DATE / POSTED DATE

$30-12-2021 / 05-01-2022$

\section{CITATION}

Madeira, Diego (2022): Necessary and Sufficient Dissipativity-Based Conditions for Feedback Stabilization. TechRxiv. Preprint. https://doi.org/10.36227/techrxiv.17707175.v1

$\mathrm{DOI}$

10.36227/techrxiv.17707175.v1 


\title{
Necessary and Sufficient Dissipativity-Based Conditions for Feedback Stabilization
}

\author{
Diego de S. Madeira
}

\begin{abstract}
Using the notion of exponential QSR-dissipativity, this work presents necessary and sufficient conditions for exponential stabilizability of nonlinear systems by linear static output feedback (SOF). It is shown that, under mild assumptions, the exponential stabilization of the closed-loop system around the origin is equivalent to the exponential QSR-dissipativity of the plant. Furthermore, whereas strict QSR-dissipativity is only sufficient for SOF asymptotic stabilization, it is proved to be necessary and sufficient for full state feedback control. New necessary and sufficient conditions for SOF stabilizability of linear systems are presented as well, along with a linear and noniterative semidefinite strategy for controller design. Necessary linear matrix inequality (LMI) conditions for stabilization are also introduced. Some examples illustrate the usefulness of the proposed approach.
\end{abstract}

Index Terms-Exponential QSR-dissipativity, exponential stability, SOF stabilizability, state feedback.

\section{INTRODUCTION}

On the realm of Lyapunov stability theory, a concept known as dissipativity, along with a special case of it called passivity, are of special interest. Primarily defined in terms of an input-output property, the notion of dissipativity was unified with the celebrated state-space perspective through the introduction of the so-called storage functions [1]. Over the last decades, this input-state-output framework has proved highly useful for asymptotic and even exponential stabilization of dynamical systems [2], [3]. Every passive system is dissipative, but the converse is not true [3]. When compared with the definition of passivity, the notion of dissipativity offers extra degrees of freedom that can be useful for controller design. Passivity applies only to square systems, while the property of dissipativity does not have this limitation [4]. Passive systems with positive definite storage functions are open-loop stable [5], whereas the class of the dissipative systems with such positive energy functions also contains open-loop unstable but feedback stabilizable plants [6].

Presently, the area of dissipativity and passivity theory constitutes a vast research topic where a number of control techniques have been proposed for feedback stabilization of linear and nonlinear systems [3], [7] - [9]. For instance, feedback passivity equivalence and global asymptotic stabilization of minimum-phase systems were handled in [10] and [11]. Feedback passivation was also discussed in details in [12] as an strategy for asymptotic stabilization of open-loop unstable systems, where the input and eventually the output of the system must be redefined in order to achieve stability. Passivity-based stabilization of nonzero equilibria was studied in [8] and [13].

In reference [14], it was shown that zero-state detectable and dissipative input-affine systems are globally asymptotically stabilizable by linear static output feedback (SOF), under certain conditions. Nevertheless, a concept known as robust linear stability was used, which is defined in terms of achieving $\dot{V} \leq-\delta h^{\top} h$ for some positive scalar $\delta$. Note that this is not the general definition of asymptotic stability $\dot{V}<0$ [15] commonly found in literature. A sufficient dissipativity-based condition for asymptotic stabilizability

Diego de S. Madeira is with the Electrical Engineering Department, Federal University of Ceará (UFC), Fortaleza 60455-760, Brazil (e-mail: dmadeira@dee.ufc.br). was proposed, which is also necessary if a certain linear growth condition on the system dynamics is fulfilled.

In [2], sufficient conditions for exponential stabilizability by output feedback were presented using the notion of exponential feedback passivity. Output feedback exponential passivity was proved equivalent to exponential minimum phaseness, whereas none of these properties are necessary for stabilization. Sufficient conditions for output feedback exponential passivity were also given in [16]. [17], on the other hand, introduced the concept of exponential dissipativity and a few sufficient conditions for open-loop and closed-loop exponential stability. Sufficient conditions for local exponential stabilization of parameterized nonlinear time-varying systems were given in [18].

Beyond the problem of equilibrium stabilization, dissipativity theory has also been applied for disturbance attenuation [19]. The socalled dissipative control problem consists in determining a control law $u=\phi(y)$ such that the closed-loop system is rendered internally stable and strictly dissipative from a disturbance $w$ to a controlled output $z$ [20]. Dissipative control comprises positive real and $H_{\infty}$ control as particular cases [20]. In article [21], output feedback of smooth nonlinear systems was handled, and state feedback was rather addressed in [22]. The dissipative control problem of LTI plants via linear SOF was addressed in [20] and [23]. Linear static state feedback and dynamic output feedback were investigated in [19].

In spite of the numerous achievements reported in the field of dissipative systems theory, many questions remain unanswered. For instance, necessary and sufficient dissipativity-based conditions for SOF exponential stabilizability of nonlinear input-affine systems still lack in the literature. Such conditions would represent an exponential stabilization counterpart to the results of [14], which addressed asymptotic stability. In the present work, these necessary and sufficient stabilizability conditions are, indeed, provided.

In this paper, the classical SOF control problem of LTI systems is also treated. This pure stabilization problem is known as a challenging one and has been approached in various ways over time [24] - [27]. Necessary and sufficient stabilizability conditions abound in the field, although they are nonlinear in nature and difficult to test. See [28] for a recent survey on this topic. In the literature, sufficient linear matrix inequality (LMI) conditions for SOF stabilizability do exist and are numerous. In the present article, though, a set of two LMIs is proved to be necessary for SOF stabilization, whereas a new related necessary and sufficient condition is rather quadratic. The internal stabilization problem $(w=0)$ is proved to be fundamentally formulated in terms of a dissipativity-based necessary and sufficient condition. A linear semidefinite programming (SDP) strategy for controller design is also proposed.

In Section II, the notation employed throughout this paper is introduced and, in Section III, a set of preliminary statements and definitions relevant to this work are presented. In Section IV, the exponential and asymptotic SOF stabilization problems of nonlinear systems are treated, as well as the SOF stabilization of LTI systems. Section V contains a solution to the state feedback control problem. Examples are given in Section VI, and Section VII contains the concluding remarks of this article and future research directions. 


\section{NOTATION}

Throughout this paper, $\mathbb{R}$ denotes the set of real numbers. $\overline{\mathbb{R}}_{+}$, $\mathbb{R}_{+}, \overline{\mathbb{R}}_{-}$and $\mathbb{R}_{-}$are, respectively, the set of elements $\beta \in \mathbb{R}$ such that $\beta \geq 0, \beta>0, \beta \leq 0$ and $\beta<0$. $\mathbb{R}^{n}$ is the set of real column vectors and $\mathbb{R}^{n \times m}$ denotes the set of $n \times m$ real matrices. $M_{i, j}$ is the $(i, j)$ element of a matrix $M, M^{\top}$ is its transpose and $M^{-1}$ is its inverse. $I$ is the identity matrix of compatible dimensions and $x_{i}$ is the $i_{t h}$ element of a vector $x \in \mathbb{R}^{n} . \mathbb{S}^{n}$ is the set of symmetric $n \times n$ matrices. $M>0(M \geq 0)$ means that $M$ is Hermitian positive definite (semidefinite), and $M<0(M \leq 0)$ means that $M$ is negative definite (semidefinite). $\|\cdot\|$ is the Euclidean norm of a vector. $f: \mathcal{X} \rightarrow \mathcal{Y}$ refers to a function $f$, a domain $\mathcal{X}$ and a codomain $\mathcal{Y} . \mathcal{X} \times \mathcal{Y}$ is the Cartesian product of sets $\mathcal{X}$ and $\mathcal{Y}$. $f \in C^{1}$ defines a (vector) function whose partial derivatives exist and are continuous, i.e. $f$ is continuously differentiable. $f: \mathcal{X} \rightarrow \mathbb{R}$, $f(0)=0$, is positive definite (semidefinite) if $f(x)>0(f(x) \geq 0)$ for any $x \in \mathcal{X}, x \neq 0 .(x, y) \in \mathcal{X}$ means that both $x$ and $y$ are elements of the same set $\mathcal{X}$ or share a common property. An open ball $\mathcal{B}_{\delta}\left(x_{e}\right)$ is defined as the set $\left\{x \in \mathbb{R}^{n}:\left\|x-x_{e}\right\|<\delta\right\}, \delta \in \mathbb{R}_{+}$.

\section{Preliminaries}

\section{A. Stability of Dynamical Systems}

Let us consider nonlinear dynamical systems with the following state-space representation [15]

$$
\dot{x}(t)=F(x(t), u(t))=f(x(t))+g(x(t)) u(t),
$$

$t \in \overline{\mathbb{R}}_{+}, x(0)=x_{0}$, where $x(t) \in \mathcal{X} \subseteq \mathbb{R}^{n}$ is the state vector and $\mathcal{X}$ is an open set with $0 \in \mathcal{X}$. Suppose that the control signal $u(t)$ is a measurable function, with $u(t) \in \mathcal{U} \subseteq \mathbb{R}^{m}$ for all $t \in \overline{\mathbb{R}}_{+}, 0 \in \mathcal{U}$. $f: \mathcal{X} \rightarrow \mathbb{R}^{n}$ and $g: \mathcal{X} \rightarrow \mathbb{R}^{n \times m}$ are such that $(f, g) \in C^{1}$, with $f(0)=0$, and the resulting $F: \mathcal{X} \times \mathcal{U} \rightarrow \mathbb{R}^{n}$ satisfies $F(0,0)=0$, i.e. the origin $(x(t), u(t)) \equiv(0,0)$ is said to be an equilibrium point of (1). $F(\cdot, \cdot)$ is continuously differentiable in a neighborhood $\mathcal{X} \times \mathcal{U}$ of the origin. In addition, a measurable output given by

$$
y(t)=h(x(t)),
$$

$h: \mathcal{X} \rightarrow \mathbb{R}^{p}, h(0)=0$, is available for feedback, where $y \in \mathcal{Y} \subseteq \mathbb{R}^{p}$ and $h \in C^{1}[15]$.

Then, consider a measurable mapping $\phi: \mathcal{X} \rightarrow \mathcal{U}$ satisfying $\phi(0)=0$. If $u(t)=u(x(t))=\phi(x(t))$ and the state $x(t)$ satisfies (1) for all $t \in \overline{\mathbb{R}}_{+}$, then $u(\cdot)$ is called a feedback control law. Suppose that the mapping $\phi(\cdot)$ satisfies sufficient regularity conditions such that the resulting closed-loop of (1)

$$
\dot{x}(t)=f(x(t))+g(x(t)) \phi(x(t))=F(x(t)),
$$

has a unique solution forward in time, $t \in \overline{\mathbb{R}}_{+}, x(0)=x_{0}$. If all state components are available and used for feedback, then $u(x)=\phi(x)$ is called a full state feedback control law [15].

Lemma 1: ( $[15$, pg. 163]) Suppose that the zero solution $x(t) \equiv 0$ to $\dot{x}(t)=F(x(t))$ in (3) is asymptotically stable, $F: \mathcal{X} \rightarrow \mathbb{R}^{n}$ fulfills $F \in C^{1}$ and let $\delta \in \mathbb{R}_{+}$be such that $\mathcal{B}_{\delta}(0) \subset \mathcal{X}$ is contained in the domain of attraction of (3). Then, there exists a function $V$ : $\mathcal{B}_{\delta}(0) \rightarrow \mathbb{R}, V \in C^{1}$, such that $V(0)=0, V(x)>0, x \in \mathcal{B}_{\delta}(0)$, $x \neq 0$, and $\dot{V}(x)=\nabla V(x)^{\top} F(x)<0, x \in \mathcal{B}_{\delta}(0), x \neq 0$.

Considering the assumptions made in Lemma 1, if a state feedback $u(x)$ asymptotically stabilizes (1) around $x(t) \equiv 0$, then

$$
\dot{V}(x)=\nabla V(x)^{\top} F(x)=\nabla V(x)^{\top}[f(x)+g(x) u(x)]<0,
$$

$x \in \mathcal{B}_{\delta}(0), x \neq 0$, for some $\delta \in \mathbb{R}_{+}$and some $V>0, V \in C^{1}$. In this paper, asymptotic feedback stabilization is always given under these conditions. The problem of linear SOF stabilization can be handled by the same framework applied for full state feedback. In this case, though, the input is restricted to the form $u(x)=u(h(x))=$ $K h(x)$, where $K \in \mathbb{R}^{m \times p}$ is a constant gain to be designed.

Finally, for $F \in C^{1}$ in (3), the exponential stability of $x(t) \equiv 0$ is verified if and only if for all $x \in \mathcal{B}_{\delta}(0)$,

$$
\begin{gathered}
\nabla V(x)^{\top}[f(x)+g(x) u(x)] \leq-\varepsilon V(x), \\
c_{1}\|x\|^{2} \leq V(x) \leq c_{2}\|x\|^{2},
\end{gathered}
$$

for $\left(\varepsilon, c_{1}, c_{2}\right) \in \mathbb{R}_{+}[15$, pg. 166], [2]. In addition,

$$
\|\nabla V(x)\| \leq c_{3}\|x\|,
$$

for some $c_{3} \in \mathbb{R}_{+}$, follows from $V \in C^{1}$ [15, pg. 247]. Throughout this work, the exponential feedback stabilization of a system means the fulfillment of (5)-(7) with some $V>0, V \in C^{1}$. Every exponentially stable equilibrium is also asymptotically stable, but the converse is only true in the case of the LTI systems [2].

\section{B. Dissipativity}

Assume that system representation (1)-(2) is completely reachable. Then, consider a supply rate $r(u(t), y(t))$ as the rate at which energy flows into the system, and a storage function $V(x)$ as the amount of energy stored inside the system. In addition, let us the supply rate be such that for all admissible $u(t)$ and $x(0)$, and for all $t \in \overline{\mathbb{R}}_{+}[15]$

$$
\int_{0}^{t}|r(u(\tau), y(\tau))| d \tau<\infty .
$$

Suppose that $V: \mathcal{X} \rightarrow \mathbb{R}$ is such that $V \in C^{1}$.

Definition 1: ( [3]) A system is said to be dissipative if there exists a storage function $V(x) \geq 0$ such that the following dissipation inequality holds

$$
\dot{V}(x) \leq r(u, y),
$$

along all possible trajectories of (1)-(2) starting at $x(0)$, for all $x(0)$, $t \in \overline{\mathbb{R}}_{+}$.

Notice that the same notation in terms of $V(x)$ used in the previous section for a Lyapunov function is applied here to an energy function. This is common in the field, as a storage function might play the role of a Lyapunov function [5]. A physical interpretation of (9) is that a dissipative system can store only a fraction of the energy supplied to it from its exterior through $r(u, y)$. Furthermore, it can deliver to its surroundings only a fraction of its stored energy $V(x)$. As the notions of storage, dissipation and transfer of energy apply to a number of practical systems, dissipativity is a key concept in many areas of control theory [15].

Definition 2: ( [3]) A dynamical system is called QSR-dissipative if it is dissipative with the following supply rate

$$
r(u, y)=y^{\top} Q y+2 y^{\top} S u+u^{\top} R u,
$$

where $Q$ and $R$ are symmetric.

Let us consider $Q \in \mathbb{S}^{p}, S \in \mathbb{R}^{p \times m}$ and $R \in \mathbb{S}^{m}$. If $Q \leq 0$ and $V(x)>0$, then the zero solution $x(t) \equiv 0$ of the uncontrolled system $(1)(u(t) \equiv 0)$ is Lyapunov stable. On the other hand, in the case of the strictly QSR-dissipative systems defined below, if $Q \leq 0$ and $V(x)>0$, the free system is asymptotically stable [15].

Definition 3: ( [3]) A system is said to be strictly QSR-dissipative if it is QSR-dissipative and there exists $T(x)>0$ such that

$$
\dot{V}+T \leq y^{\top} Q y+2 y^{\top} S u+u^{\top} R u,
$$

where $Q$ and $R$ are symmetric.

As $y=h(x)$ from (2), (11) is a function of the augmented variable $(x, u)$, and system (1)-(2) is locally strictly QSR-dissipative if this relation holds at least in a domain $(x, u) \in(\mathcal{X} \times \mathcal{U})$ around the 
point $(x(t), u(t)) \equiv(0,0)$ [29]. A sufficient matrix condition for local strict QSR-dissipativity in $\mathcal{X} \times \mathbb{R}^{m}$, i.e. for $\mathcal{U}=\mathbb{R}^{m}$, is

$$
\left[\begin{array}{cc}
\nabla V^{\top} f+T-h^{\top} Q h & \frac{1}{2} \nabla V^{\top} g-h^{\top} S \\
\left(\frac{1}{2} \nabla V^{\top} g-h^{\top} S\right)^{\top} & -R
\end{array}\right] \leq 0,
$$

for all $x \in \mathcal{X}$ [15]. If (1)-(2) is zero-state detectable, then (12) is also necessary for dissipativity with $V(x)>0$ [15].

Definition 4: ( [15]) A dynamical system is called exponentially $Q S R$-dissipative if it is strictly QSR-dissipative with $T(x)=\varepsilon V(x)$, for some $\varepsilon \in \mathbb{R}_{+}$.

If $Q \leq 0, V(x)>0$ and there exists $\left(c_{1}, c_{2}, c_{3}\right) \in \mathbb{R}_{+}$such that (6) and (7) hold, then the zero solution of the free system is exponentially stable. Every exponentially dissipative system is also strictly dissipative, the converse is not true. Lastly, another relevant special case of dissipativity is passivity, which applies only for square systems $(m=p)$. For passivity, $r(u, y)=y^{\top} u$ in (10), i.e. [15]

$$
Q=0, \quad S=\frac{1}{2} I, \quad R=0 .
$$

In order to be able to relate dissipativity to stability, the storage functions considered in this work are such that $V>0, V \in C^{1}$.

\section{Linear Static Output Feedback}

Under mild assumptions, it is possible to establish necessary and sufficient dissipativity-based conditions for linear SOF stabilizability. Both linear and nonlinear plants can be handled. In this work, stability is regarded as stability about the origin $(x(t) \equiv 0$, or simply $x=0$ ).

\section{A. The Case of Stabilizable Nonlinear Systems}

Suppose that system (1) is held exponentially stable by some linear SOF $u(x)=K h(x)$. The existence of a Lyapunov function $V(x)$ for the stable closed-loop $f(x)+g(x) u(x)$, under conditions (5)-(7), is proved to be equivalent to the exponential QSR-dissipativity of the plant representation (1)-(2) subject to (6)-(7) and a certain constraint on matrices $(Q, S, R)$. No detectability assumption is made. An early and simpler version of this result was presented in [30, pg. 83] as a sufficient condition for asymptotic stabilization. Necessity has not yet been proven.

Theorem 1: A dynamical system (1)-(2) is exponentially stabilizable by linear SOF if and only if it is exponentially QSR-dissipative with $R>0$ and $\Delta=0$, where

$$
\Delta=S R^{-1} S^{\top}-Q
$$

A stabilizing SOF is given by

$$
u=K y, \quad K=-R^{-1} S^{\top} .
$$

Proof: Necessity: Let us suppose that system (1)-(2) is exponentially stabilizable around the origin by some linear SOF $u(x)=$ $K y(x)=K h(x)$. Then, closed-loop relations (5)-(7) hold locally in an open ball $\mathcal{B}_{\delta}(0)$ for some $\left(\varepsilon, c_{1}, c_{2}, c_{3}, \delta\right) \in \mathbb{R}_{+}$and some Lyapunov function $V \in C^{1}$. From stability condition (5), there exists $\bar{\varepsilon} \in \mathbb{R}_{+}, 0<\bar{\varepsilon}<\varepsilon$, such that for $x \in \mathcal{B}_{\delta}(0), x \neq 0$,

$$
\nabla V^{\top}[f+g K h]+\bar{\varepsilon} V<0 .
$$

Consider, for instance, $\bar{\varepsilon}=\varepsilon / 2$ in (5), which leads to

$$
\nabla V^{\top}[f+g K h]+\bar{\varepsilon} V \leq-\bar{\varepsilon} V<0 .
$$

As the gradient $\nabla V$ of $V \in C^{1}$ is continuous and relation (7) holds, there exists $\beta \in \mathbb{R}_{+}$such that for all $x \in \mathcal{B}_{\delta}(0)$

$$
\nabla V^{\top}[f+g K h]+\bar{\varepsilon} V+\frac{\beta}{4} \nabla V^{\top} g g^{\top} \nabla V \leq 0,
$$

or alternatively,

$$
\nabla V^{\top} f+\bar{\varepsilon} V \leq-\nabla V^{\top} g K h-\frac{\beta}{4} \nabla V^{\top} g g^{\top} \nabla V .
$$

This statement is not trivial and demands a brief discussion. Firstly, notice that using the trace operator in (18) results in

$$
\begin{aligned}
& \nabla V^{\top}[f+g K h]+\bar{\varepsilon} V+\frac{\beta}{4} \nabla V^{\top} g g^{\top} \nabla V \\
& =\nabla V^{\top}[f+g K h]+\bar{\varepsilon} V+\frac{\beta}{4} \operatorname{tr}\left(g g^{\top} \nabla V \nabla V^{\top}\right) \\
& \leq-\bar{\varepsilon} V+\frac{\beta}{4} \operatorname{tr}\left(g g^{\top}\right) \operatorname{tr}\left(\nabla V \nabla V^{\top}\right) \\
& \leq-\bar{\varepsilon} V+\frac{\beta}{4} \operatorname{tr}\left(g^{\top} g\right)\left(\nabla V^{\top} \nabla V\right)
\end{aligned}
$$

as $\operatorname{tr}(\beta X)=\beta \operatorname{tr}(X), \operatorname{tr}(X+Y)=\operatorname{tr}(X)+\operatorname{tr}(Y), \operatorname{tr}(X Y)=$ $\operatorname{tr}(Y X)$, and if $X \geq 0$ and $Y \geq 0$, then $0 \leq \operatorname{tr}(X Y) \leq$ $\operatorname{tr}(X) \operatorname{tr}(Y)$, for any matrices $X$ and $Y$ of compatible dimensions [31]. $X$ and $Y$ might be matrix functions of the state variable such as $\nabla V \nabla V^{\top} \geq 0$ and $g^{\top} g \geq 0$. Furthermore, as $g \in C^{1}$, the real function $\operatorname{tr}\left(g^{\top} g\right) \geq 0$ is of class $C^{1}$ as well, and there exists a finite $\mathcal{G} \in \overline{\mathbb{R}}_{+}$such that $\operatorname{tr}\left(g^{\top} g\right) \leq \mathcal{G}$ for all $x \in \mathcal{B}_{\delta}(0)$ [32]. Thus, from (17), (6) and (7),

$$
\begin{aligned}
&-\bar{\varepsilon} V+\frac{\beta}{4} \operatorname{tr}\left(g^{\top} g\right)\left(\nabla V^{\top} \nabla V\right) \\
& \leq-\bar{\varepsilon} V+\beta \frac{\mathcal{G}}{4}\left(\nabla V^{\top} \nabla V\right) \\
& \leq-\bar{\varepsilon} c_{1}\|x\|^{2}+\beta\left(\frac{\mathcal{G} c_{3}^{2}}{4}\right)\|x\|^{2} .
\end{aligned}
$$

The right-hand side of relation (20) is nonpositive if

$$
-\bar{\varepsilon} c_{1}+\beta\left(\frac{\mathcal{G} c_{3}^{2}}{4}\right) \leq 0,
$$

which is feasible for

$$
0<\beta \leq \frac{4 \bar{\varepsilon} c_{1}}{\mathcal{G} c_{3}^{2}}
$$

Then, for any $\delta \in \mathbb{R}_{+}$such that $\mathcal{B}_{\delta}(0) \subset \mathcal{X}$ is contained in the domain of attraction of (1) subject to $u=K h$, there exists some $\beta \in \mathbb{R}_{+}$such that (18) and (19) are feasible for all $x \in \mathcal{B}_{\delta}(0)$.

On the other hand, suppose that dissipativity matrix condition (12) is fulfilled for all $x \in \mathcal{B}_{\delta}(0)$, with $V \in C^{1}$ such as in (6)-(7) and $T=\bar{\varepsilon} V$. This is a sufficient condition for the plant (1)-(2) to be locally exponentially QSR-dissipative. With $R>0$, (12) is feasible if and only if [15]

$$
\begin{aligned}
\nabla V^{\top} f+\bar{\varepsilon} V-h^{\top} Q h+ \\
\quad\left[\frac{1}{2} \nabla V^{\top} g-h^{\top} S\right] R^{-1}\left[\frac{1}{2} \nabla V^{\top} g-h^{\top} S\right]^{\top} \leq 0,
\end{aligned}
$$

or

$$
\begin{array}{r}
\nabla V^{\top} f+\bar{\varepsilon} V \leq h^{\top} Q h-\frac{1}{4} \nabla V^{\top} g R^{-1} g^{\top} \nabla V \\
+\frac{1}{2} \nabla V^{\top} g R^{-1} S^{\top} h+\frac{1}{2} h^{\top} S R^{-1} g^{\top} \nabla V \\
-h^{\top} S R^{-1} S^{\top} h .
\end{array}
$$

Exponential stabilizability by linear SOF implies exponential QSRdissipativity under (12) if the right-hand side of (19) is not greater 
than the right-hand side of (23), i.e. if

$$
\begin{array}{r}
-\nabla V^{\top} g K h-\frac{\beta}{4} \nabla V^{\top} g g^{\top} \nabla V \leq h^{\top} Q h \\
-\frac{1}{4} \nabla V^{\top} g R^{-1} g^{\top} \nabla V+\frac{1}{2} \nabla V^{\top} g R^{-1} S^{\top} h \\
+\frac{1}{2} h^{\top} S R^{-1} g^{\top} \nabla V-h^{\top} S R^{-1} S^{\top} h .
\end{array}
$$

Assume $R=\frac{1}{\beta} I>0$, without loss of generality, where $\beta$ comes from (21). Thus, (19) implies (23) if

$$
\begin{gathered}
-\nabla V^{\top} g K h-\frac{1}{4} \nabla V^{\top} g R^{-1} g^{\top} \nabla V \leq h^{\top} Q h \\
-\frac{1}{4} \nabla V^{\top} g R^{-1} g^{\top} \nabla V+\frac{1}{2} \nabla V^{\top} g R^{-1} S^{\top} h \\
+\frac{1}{2} h^{\top} S R^{-1} g^{\top} \nabla V-h^{\top} S R^{-1} S^{\top} h,
\end{gathered}
$$

which can be rearranged into

$$
\nabla V^{\top} g\left[K+R^{-1} S^{\top}\right] h-h^{\top} \Delta h \geq 0
$$

As $R=\frac{1}{\beta} I$, the stabilizing gain $K$ can always be decomposed in terms of $R$ and $S$ in the following way

$$
K=-R^{-1} S^{\top}
$$

where $S$ is set according to

$$
S=-K^{\top} R .
$$

By applying (26), inequality (25) is now given by

$$
-h^{\top} \Delta h \geq 0,
$$

which is fulfilled if $\Delta \leq 0$. $Q$ is a free parameter and $\Delta=0$ is obtained by setting $Q=S R^{-1} S^{\top}$. This means that if (1)-(2) has a linear SOF stabilizable equilibrium at the $x=0$, then it is exponentially QSR-dissipative in a domain $(x, u) \in\left(\mathcal{B}_{\delta}(0) \times \mathbb{R}^{m}\right)$ with $R>0$ and $\Delta=0$, as it fulfills sufficient condition (12).

Sufficiency: With $T=\varepsilon V$ in (11), one has the definition of exponential QSR-dissipativity of a system

$$
\nabla V^{\top}[f+g u]+\varepsilon V \leq y^{\top} Q y+2 y^{\top} S u+u^{\top} R u .
$$

For $y=h,(28)$ is a function of $x$ and $u$ and holds, by assumption, in a region $(x, u) \in(\mathcal{X} \times \mathcal{U})$ around $(x, u)=(0,0)$. The right-hand side of (28) consists in the supply rate presented in (10) and note that $r(u, y) \geq 0$ if

$$
\left[\begin{array}{cc}
Q & S \\
S^{\top} & R
\end{array}\right] \geq 0
$$

which is equivalent to

$$
R>0, \quad Q-S R^{-1} S^{\top}=-\Delta \geq 0
$$

Next, let us suppose that system (1)-(2) is exponentially QSRdissipative with $R>0$ and $\Delta=0(r(u, y) \geq 0)$ in (28). Our problem consists, then, in investigating whether there exists a linear SOF $u=$ $K y=K h$ such that this dissipative system is held exponentially stable around the origin, by fulfilling condition (5) with a positive definite function $V \in C^{1}$ that also verifies (6) and (7) in a set $\mathcal{X} \supset 0$.

The answer to this question appears by analyzing the necessary conditions for a differentiable function $r(u, y) \geq 0$ to attain its minimum whose value is zero. If some control signal minimizes $r(u, y)$, then (28) is reduced to $\nabla V^{\top}[f+g u]+\varepsilon V \leq 0$, which guarantees exponential stability. The aforementioned necessary optimality conditions amount to

$$
\nabla r=\left[\begin{array}{l}
r_{u} \\
r_{y}
\end{array}\right]=\left[\begin{array}{c}
2 R u+2 S^{\top} y \\
2 Q y+2 S u
\end{array}\right]=\left[\begin{array}{l}
0 \\
0
\end{array}\right],
$$

where $r_{u}$ and $r_{y}$ are the partial derivatives of the supply rate with respect to $u$ and $y$, respectively. A linear SOF given by (15) and a set of matrices $(Q, S, R)$ such that $\Delta=0$ solve (31). It minimizes the supply rate by resulting in $r(u, y)=0$, for all $x \in \mathcal{X}$, as

$$
\min r(u, y)=r(K y, y)=-y^{\top} \Delta y=0 \text {. }
$$

Again, from (28), $\Delta=0$ implies

$$
\nabla V^{\top}[f+g u]+\varepsilon V \leq 0 \Rightarrow \nabla V^{\top}[f+g u] \leq-\varepsilon V,
$$

$x \in \mathcal{X}$, which is the same as (5). This means that the origin is exponentially stable and $\Delta=0$ is also a sufficient condition for stabilization. In conclusion, a system is exponentially stabilizable by linear SOF if and only if it is exponentially QSR-dissipative with $R>0$ and $\Delta=0$.

It is important to stress that fulfilling relation (28) alone does not guarantee stability, as it simply sets an upper bound for $\dot{V}+\varepsilon V$ in $(\mathcal{X} \times \mathcal{U})$. Exponential stability is obtained if $\Delta=0$ and the control signal assumes the particular form $u=-R^{-1} S^{\top} h$.

Lastly, a version of Theorem 1 can be presented in terms of a sufficient condition for asymptotic stabilization. In the following corollary, $V>0$ is again supposed to be continuously differentiable, whereas the assumption of relations (5)-(7) is not made. Instead, condition (4) is applied, which is the definition of asymptotic stability in closed-loop.

Corollary 1: Suppose that $R>0$ and $\Delta$ is given by (14). If a dynamical system (1)-(2) is strictly QSR-dissipative with $\Delta \geq 0$, then it is asymptotically stabilizable by the linear SOF (15).

Proof: Under (14) and (15), strict QSR-dissipativity condition (11) results in

$$
\nabla V^{\top}[f+g K h]+T \leq-h^{\top} \Delta h .
$$

If $\Delta \geq 0$, with $h(0)=0$, then the origin is asymptotically stable due to (4). Furthermore, as $\Delta \geq 0$, there exists some $Q_{2} \geq Q$ such that $\Delta_{2}=S R^{-1} S^{\top}-Q_{2}=0$, which guarantees stability as well.

Remark 1: In the case of systems (1)-(2), strict QSR-dissipativity could not be established as a necessary condition for asymptotic stabilization. For, from (18), it would be necessary to prove the existence of some $\beta \in \mathbb{R}_{+}$such that

$$
\nabla V^{\top}[f+g K h]+T+\frac{\beta}{4} \nabla V^{\top} g g^{\top} \nabla V \leq 0,
$$

without considering conditions (5)-(7). This has not been proved.

\section{B. The Stabilization Problem of LTI Systems}

In the LTI case, asymptotic and exponential stability are known to be equivalent [2] and dissipativity-based conditions for stabilizability can be presented in the much convenient form of LMIs and SDP problems. Thus, they can be solved very efficiently with widely available softwares [33] - [35].

Firstly, consider a continuous-time LTI system

$$
\dot{x}=A x+B u, \quad y=C x,
$$

where $x \in \mathbb{R}^{n}, u \in \mathbb{R}^{m}, y \in \mathbb{R}^{p}, A \in \mathbb{R}^{n \times n}, B \in \mathbb{R}^{n \times m}$ and $C \in \mathbb{R}^{p \times n}$. By applying the following linear SOF

$$
u=K y, \quad K \in \mathbb{R}^{m \times p},
$$

the closed-loop system is given by

$$
\dot{x}=(A+B K C) x .
$$


Lemma 2: ( [27]) A static gain $K$ stabilizes (38) if and only if there exists a symmetric matrix $P>0$ such that

$$
(A+B K C)^{\top} P+P(A+B K C)<0 .
$$

The SOF control problem (39) is not convex and presents nondifferentiability issues [28]. As a result, it is hard to solve numerically, and a simple, testable, and at the same time necessary and sufficient condition is not known. In the following, dissipativity theory is applied to this problem.

Lemma 3: ( [15]) The LTI plant (36) is strictly QSR-dissipative if the following LMI is fulfilled

$$
\left[\begin{array}{cc}
\left(A^{\top} P+P A+N-C^{\top} Q C\right) & \left(P B-C^{\top} S\right) \\
\left(P B-C^{\top} S\right)^{\top} & -R
\end{array}\right] \leq 0,
$$

for some $S$ and for symmetric matrices $Q, R$ and $N>0$.

An adaptation of Theorem 1 to the LTI case is given below. An early version of this result can be found in [30, pg. 59].

Theorem 2: The LTI system (36) is linear SOF stabilizable if and only if (40) is feasible with $(R, P, N)>0$ and $\Delta=0$, where

$$
\Delta=S R^{-1} S^{\top}-Q \text {. }
$$

A stabilizing gain is given by

$$
K=-R^{-1} S^{\top} \text {. }
$$

Proof: Necessity: Let system (36) be SOF stabilizable. Then, there exist $P>0$ and $K$ such that (39) holds. As a consequence, there exists $N>0, N \in \mathbb{R}^{n \times n}$, that fulfills

$$
(A+B K C)^{\top} P+P(A+B K C)+N<0,
$$

as one can set $N=-\eta\left[(A+B K C)^{\top} P+P(A+B K C)\right]>0$, $0<\eta<1$. From Appendix I, there exists $\beta \in \mathbb{R}_{+}$such that

$$
(A+B K C)^{\top} P+P(A+B K C)+N+\beta P B B^{\top} P \leq 0,
$$

which is equivalent to

$$
\begin{aligned}
& A^{\top} P+P A+N \leq \\
& \quad-C^{\top} K^{\top} B^{\top} P-P B K C-\beta P B B^{\top} P .
\end{aligned}
$$

Next, recover that dissipativity condition (40) holds with $R=$ $\mu I>0, \mu=\frac{1}{\beta}$, if and only if

$$
\begin{aligned}
& \left(A^{\top} P+P A+N-C^{\top} Q C\right)+ \\
& \quad \beta\left(P B-C^{\top} S\right)\left(P B-C^{\top} S\right)^{\top} \leq 0 .
\end{aligned}
$$

Again, without loss of generality, $R=\frac{1}{\beta} I$. After reorganizing (46),

$$
\begin{array}{r}
A^{\top} P+P A+N \leq C^{\top} Q C-\beta C^{\top} S S^{\top} C+ \\
\beta P B S^{\top} C+\beta C^{\top} S B^{\top} P-\beta P B B^{\top} P .
\end{array}
$$

Then, stabilizability by SOF implies the feasibility of (40) if the righthand side of (45) is not greater than the expression on the right-hand side of (47), i.e. if

$$
\begin{array}{r}
-C^{\top} K^{\top} B^{\top} P-P B K C-\beta P B B^{\top} P \leq C^{\top} Q C \\
-\beta C^{\top} S S^{\top} C+\beta P B S^{\top} C+\beta C^{\top} S B^{\top} P-\beta P B B^{\top} P,
\end{array}
$$

which is satisfied if

$$
\begin{array}{r}
P B\left(K+\beta S^{\top}\right) C+C^{\top}\left(K+\beta S^{\top}\right)^{\top} B^{\top} P+ \\
C^{\top}\left(Q-\beta S S^{\top}\right) C \geq 0 .
\end{array}
$$

Now, set

$$
K=-\beta S^{\top}=-R^{-1} S^{\top} \Rightarrow S=-\frac{1}{\beta} K^{\top}=-\mu K^{\top}
$$

Relation (49) is, then, reduced to

$$
C^{\top}\left(Q-\mu K^{\top} K\right) C=C^{\top}\left(Q-S R^{-1} S^{\top}\right) C=-C^{\top} \Delta C \geq 0,
$$

and this inequality holds if $Q-\mu K^{\top} K \geq 0$, i.e. if $\Delta \leq 0$. Again, $\Delta=0$ is obtained by setting $Q=S R^{-1} S^{\top}$. Thus, (36) is SOF stabilizable only if (40) is feasible with $(R, P, N)>0$ and $\Delta=0$.

Sufficiency: Suppose that (40) is feasible for some $(Q, S)$ and $(R, P, N)>0$, which guarantees that (36) is strictly QSRdissipative. With $R>0$, LMI (40) holds if and only if

$$
\begin{array}{r}
\left(A^{\top} P+P A+N-C^{\top} Q C\right)+P B R^{-1} B^{\top} P \\
-P B R^{-1} S^{\top} C-C^{\top} S R^{-1} B^{\top} P+C^{\top} S R^{-1} S^{\top} C \leq 0,
\end{array}
$$

Applying (42) leads to

$$
\begin{array}{r}
(A+B K C)^{\top} P+P(A+B K C) \leq \\
-P B R^{-1} B^{\top} P-N-C^{\top} \Delta C .
\end{array}
$$

If $\Delta \geq 0$, (39) certainly holds and the system is stabilizable. Thus, the feasibility of (40) with $(R, P, N)>0$ and $\Delta=0$ is a necessary and sufficient condition for asymptotic stabilizability by linear SOF.

Once a solution $(P, N, Q, S, R)$ for (40) with $\Delta=0$ is found, the combination $(\delta P, \delta N, \delta Q, \delta S, \delta R)$ with any $\delta \in \mathbb{R}_{+}$also solves (40) resulting in the same gain $K$ and the same $\Delta$. Then, without loss of generality, one can consider $R=I$.

Yet, the necessary condition of having $\Delta \leq 0$ with $R>0(R=I)$ is equivalent to a simple LMI, as

$$
\left\{\begin{array}{l}
R>0 \\
\Delta=S R^{-1} S^{\top}-Q \leq 0
\end{array} \Leftrightarrow X_{d}=\left[\begin{array}{cc}
Q & S \\
S^{\top} & R
\end{array}\right] \geq 0,\right.
$$

with $\operatorname{tr}\left(X_{d}\right) \geq 0$. As a result, one can formulate the following linear SDP problem for controller design,

$$
\begin{aligned}
\text { minimize } & \operatorname{tr}\left(X_{d}\right), \\
\text { subject to } & X_{d} \geq 0,(40),
\end{aligned}
$$

$\operatorname{tr}\left(X_{d}\right)=0 \Leftrightarrow X_{d}=0$ [36]. By solving optimization problem (54), one guarantees that the necessary condition $\Delta \leq 0$ is fulfilled and, at the same time, tries to come as close as possible to the necessary and sufficient constraint that $\Delta=0$. With $R=I$, the objective function $\operatorname{tr}\left(X_{d}\right)$ is minimized by some triple $(Q, S, R)$ to which $\Delta=0$, although the converse is not true. A gain $K$ is given by (42).

Remark 2: Note that a necessary LMI condition for SOF stabilizability is given by simultaneously fulfilling $X_{d} \geq 0$ and (40).

The results of Theorem 2 are equivalent to the necessary and sufficient conditions proposed in [37] in a resilient approach for control, although no mention or use of dissipativity theory is made in that interesting reference. In [37], the authors followed a different approach and did not base their work in linear SDP formulations such as (54), rather solving the SOF control problem in a nonlinear and iterative fashion. No necessary LMI condition was given either.

An alternative formulation which may result in the feasibility of linear SDP problem (54) with $\Delta>0$ consists in the following strategy. Consider some $\alpha \in \mathbb{R}_{+}$and define a new $X_{d}$ as

$$
X_{d}=\left[\begin{array}{cc}
Q+\alpha I & S \\
S^{\top} & R
\end{array}\right] \geq 0,
$$

which is equivalent to

$$
\left\{\begin{array}{l}
R>0 \\
\Delta=S R^{-1} S^{\top}-Q \leq \alpha I .
\end{array}\right.
$$


Then, solve (54) with $X_{d}$ as given in (55). By minimizing $\operatorname{tr}\left(X_{d}\right)$ one might approach the positive upper bound of $\Delta=\alpha I>0$. Without loss of generality, one can consider $\alpha=1$. Besides, as stated in the following corollary, $\Delta>0$ is not only sufficient but also necessary to stabilization. An example in Section VI-B shows how that can be obtained simultaneously to (40) via semidefinite programming.

Corollary 2: The LTI system (36) is linear SOF stabilizable if and only if (40) is feasible with $(R, P, N)>0$ and $\Delta>0$, where $\Delta$ is given by (41). A stabilizing gain is given by (42).

Proof: Necessity: If the plant is stabilizable, then (52) is feasible with $\Delta=S R^{-1} S^{\top}-Q=0$ and the right-hand side of this inequality is equal to $-P B R^{-1} B^{\top} P-N<0$. By setting a new $Q_{2}=Q-\gamma I$, for some $\gamma \in \mathbb{R}_{+}$it results in $\Delta_{2}=S R^{-1} S^{\top}-Q_{2}=\gamma I>0$, which still verifies (52) if $N$ is redefined as $N_{2}=N-\gamma C^{\top} C>0$. From Appendix I, there is a $\gamma \in \mathbb{R}_{+}$small enough such that $-N_{2}<0\left(N_{2}>0\right)$.

Sufficiency: It follows directly from (52).

\section{State Feedback Stabilization}

From a dissipativity-based perspective, the problem of state feedback asymptotic stabilization of (1) consists in specifying a suitable (fictitious) output variable (2) for the plant, in such a manner that the resulting representation (1)-(2) is rendered SOF stabilizable according to the framework of Corollary 1. Nevertheless, in a state feedback scenario, strict QSR-dissipativity is proved not only sufficient, but also necessary for asymptotic stabilization. In [30, pg. 83], a similar result was presented as a sufficient condition.

Theorem 3: A dynamical system (1) is asymptotically stabilizable by state feedback if and only if there exists an (fictitious) output variable (2) such that the system representation (1)-(2) is strictly QSR-dissipative with $R>0$ and $\Delta=0$, where

$$
\Delta=S R^{-1} S^{\top}-Q .
$$

A stabilizing state feedback is given by

$$
u(x)=-R^{-1} S^{\top} h(x) .
$$

Proof: Necessity: If (1) is asymptotically stabilizable by some state feedback $u(x)$, then condition (4) holds in an open ball $\mathcal{B}_{\delta}(0) \subset$ $\mathcal{X}$. This means that there exists a function $T: \mathcal{B}_{\delta}(0) \rightarrow \mathbb{R}(T(0)=$ $0, T(x)>0, x \neq 0)$, such that for all $x \in \mathcal{B}_{\delta}(0)$,

$$
\nabla V^{\top}[f+g u]+T \leq 0 .
$$

Furthermore, for any $\beta \in \mathbb{R}_{+}$the following relation holds in $\mathcal{B}_{\delta}(0)$,

$$
\nabla V^{\top}[f+g \bar{u}]+T+\frac{\beta}{4} \nabla V^{\top} g g^{\top} \nabla V \leq 0,
$$

where

$$
\bar{u}(x)=u(x)-\frac{\beta}{4} g(x)^{\top} \nabla V(x) .
$$

Then, by defining an output

$$
h(x)=\bar{u}(x),
$$

one recovers $\bar{u}=K y=K h$ with $K=I$. By setting $R=\frac{1}{\beta} I>0$, (58) holds if

$$
-R^{-1} S^{\top}=I \Rightarrow S=-\frac{1}{\beta} I
$$

As in Theorem 1, stability implies dissipativity if (24) and (25) are verified, which is the case if $K=-R^{-1} S^{\top}$ and $\Delta=0$ (set $Q=S R^{-1} S^{\top}$ ). Thus, stabilizability by state feedback implies strict QSR-dissipativity with $R>0$ and $\Delta=0$, with the output (62).

Sufficiency: It follows directly from Corollary 1 . If all state variables are measured and used for control, then $u(x)$ is a state feedback control law.

In general, the results of sections IV and V could not be established using the notion of passivity, since in this case $R=0$ and $Q=0$.

\section{EXAMPLES}

\section{A. Nonlinear Model of a Microelectromechanical System}

Let us consider the normalized model of a micro-electromechanical system (MEMS) valid locally around the origin [38],

$$
\dot{x}=\left[\begin{array}{c}
\dot{x}_{1} \\
\dot{x}_{2} \\
\dot{x}_{3}
\end{array}\right]=\left[\begin{array}{c}
x_{2} \\
-x_{1}-2 \xi x_{2}+\frac{2 \bar{q}}{3} x_{3}+\frac{1}{3} x_{3}^{2} \\
\frac{1}{r} \bar{q} x_{1}+\frac{1}{r} x_{1} x_{3}+\frac{(\bar{x}-1)}{r} x_{3}
\end{array}\right]+\left[\begin{array}{c}
0 \\
0 \\
\frac{2}{3 r}
\end{array}\right] u,
$$

where $(\bar{q}, \xi, r) \in \mathbb{R}_{+},(\bar{x}-1) \in \mathbb{R}_{-}$. Here, $x_{1}$ is the plate deflection, $x_{2}$ is the deflection speed and $x_{3}$ is the charge. Suppose that system (64) must be stabilized about $x=0$ by measuring the charge only,

$$
y(x)=h(x)=x_{3} .
$$

Strict dissipativity condition (12) holds locally for some $T>0$ and $R>0$ if, for example,

$$
\begin{gathered}
\frac{1}{2} \nabla V^{\top} g=h^{\top} S, \\
\nabla V^{\top} f-h^{\top} Q h<0,
\end{gathered}
$$

with $(Q, S, R) \in \mathbb{R}$. As $\nabla V=\left[\begin{array}{lll}V_{x_{1}} & V_{x_{2}} & V_{x_{3}}\end{array}\right]^{\top}$, (66) gives

$$
\frac{1}{2}\left[\begin{array}{lll}
V_{x_{1}} & V_{x_{2}} & V_{x_{3}}
\end{array}\right]\left[\begin{array}{c}
0 \\
0 \\
\frac{2}{3 r}
\end{array}\right]=x_{3} S \Rightarrow V_{x_{3}}=3 S r x_{3} .
$$

This means by direct integration that

$$
V\left(x_{1}, x_{2}, x_{3}\right)=\frac{3 S r}{2} x_{3}^{2}+V_{1}\left(x_{1}, x_{2}\right),
$$

where $S>0$ is mandatory for $V>0$. Now, (67) needs to be solved,

$$
\begin{array}{r}
x_{2} V_{x_{1}}+\left(-x_{1}-2 \xi x_{2}+\frac{2 \bar{q}}{3} x_{3}+\frac{1}{3} x_{3}^{2}\right) V_{x_{2}} \\
+\left(\frac{1}{r} \bar{q} x_{1}+\frac{1}{r} x_{1} x_{3}+\frac{(\bar{x}-1)}{r} x_{3}\right)\left(3 S r x_{3}\right)-Q x_{3}^{2}<0 .
\end{array}
$$

By considering

$$
V_{x_{1}}=v_{1} x_{1}+v_{3} x_{2}, \quad V_{x_{2}}=v_{2} x_{2}+v_{3} x_{1},
$$

with $\left(v_{1}, v_{2}, v_{3}\right) \in \mathbb{R}_{+}$, it results by integration in

$$
V\left(x_{1}, x_{2}, x_{3}\right)=\frac{v_{1}}{2} x_{1}^{2}+\frac{v_{2}}{2} x_{2}^{2}+\frac{3 S r}{2} x_{3}^{2}+v_{3} x_{1} x_{2},
$$

and (70) is now given by

$$
\begin{aligned}
v_{1} x_{1} x_{2}+v_{3} x_{2}^{2}-v_{2} x_{1} x_{2}-2 \xi v_{2} x_{2}^{2}+\frac{2 \bar{q} v_{2}}{3} x_{2} x_{3}+\frac{v_{2}}{3} x_{2} x_{3}^{2} \\
+3 S \bar{q} x_{1} x_{3}+3 S x_{1} x_{3}^{2}+3 S(\bar{x}-1) x_{3}^{2}-Q x_{3}^{2}-v_{3} x_{1}^{2} \\
-2 \xi v_{3} x_{1} x_{2}+\frac{2 \bar{q} v_{3}}{3} x_{1} x_{3}+\frac{v_{3}}{3} x_{1} x_{3}^{2}<0
\end{aligned}
$$

Then, define a function $N_{3}(x)$ containing all terms of order higher than two,

$$
N_{3}(x)=\frac{v_{2}}{3} x_{2} x_{3}^{2}+3 S x_{1} x_{3}^{2}+\frac{v_{3}}{3} x_{1} x_{3}^{2} .
$$

As a result, (73) is equivalent to

$$
\begin{aligned}
& D(x)=-v_{3} x_{1}^{2}+\left(v_{3}-2 \xi v_{2}\right) x_{2}^{2}+[3 S(\bar{x}-1)-Q] x_{3}^{2} \\
&+\left(v_{1}-v_{2}-2 \xi v_{3}\right) x_{1} x_{2}+\frac{2 \bar{q} v_{2}}{3} x_{2} x_{3} \\
&+\left(3 S \bar{q}+\frac{2 \bar{q} v_{3}}{3}\right) x_{1} x_{3}+N_{3}(x) .
\end{aligned}
$$


At the origin, the Hessian matrix of $D(x)\left(\nabla^{2} D\right)$ is not affected by the terms of third order in $N_{3}(x)$, as their second order derivatives vanish at this point. Then, by setting

$$
v_{1}=v_{2}+2 \xi v_{3},
$$

$\left.\nabla^{2} D\right|_{x=0}$ is given by

$$
\left[\begin{array}{ccc}
-2 v_{3} & 0 & \left(3 S \bar{q}+\frac{2 \bar{q} v_{3}}{3}\right) \\
0 & 2 v_{3}-4 \xi v_{2} & \frac{2 \bar{q}}{3} v_{2} \\
\left(3 S \bar{q}+\frac{2 \bar{q} v_{3}}{3}\right) & \frac{2 \bar{q}}{3} v_{2} & 2[3 S(\bar{x}-1)-Q]
\end{array}\right] .
$$

Next, consider

$$
S=-\frac{(\bar{x}-1)}{3}>0
$$

and define

$$
\gamma=2\left[(\bar{x}-1)^{2}+Q\right]>0,
$$

with $Q>0$. Then, $\left.\nabla^{2} D\right|_{x=0}<0$ if and only if

$$
\begin{aligned}
& {\left[\begin{array}{cc}
-2 v_{3} & 0 \\
0 & v_{3}-4 \xi v_{2}
\end{array}\right]} \\
& +\left[\begin{array}{c}
\left(3 S \bar{q}+\frac{2 \bar{q} v_{3}}{3}\right) \\
\frac{2 \bar{q}}{3} v_{2}
\end{array}\right](\gamma)^{-1}\left[\begin{array}{c}
\left.3 S \bar{q}+\frac{2 \bar{q} v_{3}}{3}\right) \\
\frac{2 \bar{q}}{3} v_{2}
\end{array}\right]^{\top}<0 .
\end{aligned}
$$

For some $v_{2}$ and for sufficiently small $v_{3}$ such that

$$
\left[\begin{array}{cc}
-2 v_{3} & 0 \\
0 & v_{3}-4 \xi v_{2}
\end{array}\right]<0
$$

(80) is valid if and only if $Q$ is large enough, i.e. if $\gamma^{-1}$ is sufficiently small (Appendix I also explains why this is the case).

Furthermore, for $v_{3}$ sufficiently small, the energy function $V(x)$ is locally positive definite, as $\left.\nabla^{2} V\right|_{x=0}>0$ can be obtained regardless of $Q$ and $R$. The remaining parameter $v_{1}$ is determined according to (76). Finally, asymptotic stability is obtained by fixing $\Delta=S R^{-1} S^{\top}-Q=0$, for some sufficiently small $R>0$ that can also be set independently. A stabilizing gain is finally given by $u=-R^{-1} S^{\top} x_{3}$. Due to the successful tests with the Hessian matrices $\nabla^{2} V>0$ and $\nabla^{2} D<0$, the origin is also exponentially stable, as there exist $\left(\varepsilon, c_{1}, c_{2}\right) \in \mathbb{R}_{+}$such that (5)-(6) hold. This is the case because $V(x)$ and $D(x)$ are polynomials, thus continuously differentiable and locally bounded by $\|x\|^{2}$ functions.

The main advantage of this dissipativity-based control technique is that it offers more degrees of freedom than passivity-based strategies, since matrices $(Q, S, R)$ might be manipulated with great flexibility in order to achieve stability. Moreover, dissipativity is a necessary and sufficient condition for SOF exponential stabilization, which is not the case of passivity. Lastly, it does not demand any fixed closedloop dynamics such as a port-controlled Hamiltonian structure, for example, in order to have feedback stabilization.

\section{B. An LTI System}

In this example of SOF design using the framework of Theorem 2, we deal with the $A C 4$ model of [39], which is open-loop unstable

$$
\begin{gathered}
A=\left[\begin{array}{cccc}
-0.876 & 1 & -0.1209 & 0 \\
8.9117 & 0 & -130.75 & 0 \\
0 & 0 & -150 & 0 \\
-1 & 0 & 0 & -0.05
\end{array}\right], \\
B=\left[\begin{array}{llll}
0 & 0 & 150 & 0
\end{array}\right]^{\top}, \quad C=\left[\begin{array}{cccc}
-1 & 0 & 0 & 0 \\
0 & -1 & 0 & 0
\end{array}\right] .
\end{gathered}
$$

Next, consider $X_{d}$ as in (55), $\alpha=R=1$ and $P>10^{-2} I$. By solving (54) using semidefinite programming [34] - [35], we obtain

$$
\begin{gathered}
Q=\left[\begin{array}{cc}
-0.8729 & 0.4229 \\
0.4229 & 0.4149
\end{array}\right], \quad S=\left[\begin{array}{l}
0.1931 \\
0.7149
\end{array}\right], \\
\Delta=\left[\begin{array}{cc}
0.9102 & -0.2849 \\
-0.2849 & 0.0962
\end{array}\right]>0, \quad K=\left[\begin{array}{ll}
-0.1931 & -0.7149
\end{array}\right],
\end{gathered}
$$

and the closed-loop poles: $-0.0500,-74.9072 \pm j 91.5283,-1.0615$.

This simple example illustrates the usefulness of the results introduced in Section IV-B. Program (54) is a linear semidefinite strategy which is capable of solving the challenging SOF control problem in a noniterative fashion, as opposed to other strategies known in the field [28]. QSR-dissipativity is not only fundamentally related to the SOF problem, its application also leads to a simple linear SDP procedure for controller design.

\section{Conclusion and Future Research}

In this work, it was shown that the exponential stabilizability of input-affine nonlinear systems by linear SOF is, under a few assumptions, equivalent to the exponential QSR-dissipativity of the plant. This necessary and sufficient result holds for local stabilization and can be extended for state feedback of nonlinear plants and SOF control of LTI systems. On the other hand, strict dissipativity is only sufficient for asymptotic stabilization via linear SOF. The focus on dissipativity matrices $(Q, S, R)$ and the $\Delta$ variable enables the formulation of a simple linear SDP program for SOF control of LTI plants. Finally, a set of two dissipativity-based LMIs are proved to be necessary for stabilization of linear systems. To the best of my knowledge, all these results are new in the field of dissipativity theory.

As discussed in [30], formulation (54) combined with the Finsler's Lemma and a polytopic LMI approach can be used for control of rational plants. This is an interesting direction of ongoing and also of future research, as well as the case of systems with input saturation. Preliminary results of a linear SDP approach for local robust stabilization are available in [40]. Another subject of forthcoming investigation involves the application of Theorem 1 and some $\Delta \geq 0$ condition to equilibrium-independent dissipative systems [41] and the feedback stabilization problem of nonzero equilibria.

Finally, a future research topic must be a detailed comparison between the strategy presented in Section IV-B and those already known in the literature [42] - [44]. An application of the method for a wide group of benchmark plants such as [39] is still needed.

\section{APPENDIX I}

Theorem 4: Let us consider real symmetric and constant matrices $X$ and $Y$ of suitable dimensions and let us define a matrix function

$$
M(\gamma)=X+\gamma Y, \quad X<0, \quad Y>0, \quad \gamma \in \overline{\mathbb{R}}_{+} .
$$

Then, there exists some $\gamma \in \mathbb{R}_{+}$such that $M(\gamma) \leq 0$.

Proof: Firstly, the highest eigenvalue $\lambda_{\max }$ of the real matrix $M(\gamma)$ is a real continuous function of its entries, although it might not be smooth [31]. Actually, $\lambda_{\max }(M(\gamma))$ is a function of $\gamma$, where

$$
\lambda_{\max }(M(0))=\lambda_{\max }(X)<0 .
$$

As $\lambda_{\max }(M(\gamma))$ is continuous on $\gamma$, there exists some $\gamma_{1} \in \mathbb{R}_{+}$ such that $\lambda_{\max }\left(M\left(\gamma_{1}\right)\right)$ is still strictly negative and $M\left(\gamma_{1}\right)<0$. This is true even if $Y \geq 0$ (only negative semidefinite).

Furthermore, there also exists some $\gamma_{2} \in \mathbb{R}_{+}$such that $\lambda_{\max }\left(M\left(\gamma_{2}\right)\right)=0$ exactly, which implies $M\left(\gamma_{2}\right) \leq 0$. This result is obtained by applying the so-called Cauchy Interlacing Law [45, pg. 396], which affirms that

$$
\lambda_{\max }(M(\cdot)) \geq M_{1,1}(\cdot)
$$


where $M_{1,1}(\cdot)$ is the element at the upper left corner of $M(\cdot)$. As

$$
M_{1,1}(\gamma)=X_{1,1}+\gamma Y_{1,1},
$$

where $X_{1,1}<0$ and $Y_{1,1}>0$, one has $M_{1,1}(\cdot)>0$ for

$$
\gamma_{2}>\frac{-X_{1,1}}{Y_{1,1}}>0
$$

This means that for any $\gamma_{2} \in \mathbb{R}_{+}$such that (86) holds, $\lambda_{\max }\left(M\left(\gamma_{2}\right)\right)>0$. Finally, from the Intermediate Value Theorem [46], there exists some $\gamma_{3} \in \mathbb{R}_{+}$such that $0<\gamma_{1} \leq \gamma_{3} \leq \gamma_{2}$ and $\lambda_{\max }\left(M\left(\gamma_{3}\right)\right)=0 \Rightarrow M\left(\gamma_{3}\right) \leq 0$. This reasoning had also been used in [47] for solving a problem in the field of passivity theory.

\section{REFERENCES}

[1] J. L. Willems, "A system theory approach to unified electrical machine analysis," Int. J. of Control, vol. 15, no. 3, pp. 401-418, Mar. 1972.

[2] A. Fradkov and D. J. Hill, "Exponential feedback passivity and stabilizability of nonlinear systems," Automatica, vol. 34, no. 6, pp. 697-703, Jun. 1998.

[3] B. Brogliato, R. Lozano, B. Maschke, and O. Egeland, Dissipative Systems Analysis and Control - Theory and Applications. London, UK Springer-Verlag, 2020.

[4] J. C. Willems, "Dissipative dynamical systems - part II: Linear systems with quadratic supply rates," Arch. for Rational Mech. Anal., vol. 45, pp. 352-393, 1972.

[5] H. K. Khalil, Nonlinear Systems. Upper Saddle River, NJ, USA: Prentice Hall, 2002.

[6] D. J. Hill and P. J. Moylan, "The stability of nonlinear dissipative systems," IEEE Trans. Autom. Control, vol. 21, no. 5, pp. 708-711, Oct. 1976.

[7] W. M. Haddad and D. S. Bernstein, "Robust stabilization with positive real uncertainty: Beyond the small gain theorem," in Proc. 29th IEEE Conf. Decis. Control, Honolulu, HI, USA, 1990, pp. 2054-2059.

[8] R. Ortega, J. A. Loria Perez, P. J. Nicklasson and H. Sira-Ramirez, Passivity-based Control of Euler-Lagrange Systems - Mechanical, Electrical and Electromechanical Applications. London, UK: SpringerVerlag, 1998.

[9] D. J. Hill and P. J. Moylan, "Stability results for nonlinear feedback systems," Automatica, vol. 13, no. 4, pp. 377-382, Jul. 1977.

[10] P. V. Kokotovic and H. J. Sussmann, "A positive real condition for globa stabilization of nonlinear systems," Syst. Control Lett., vol. 13, no. 2, pp. 125-133, Aug. 1989.

[11] C. I. Byrnes, A. Isidori, and J. C. Willems, "Passivity, feedback equivalence and global stabilization of minimum phase nonlinear systems," IEEE Trans. Autom. Control, vol. 36, no. 11, pp. 1228-1240, Nov. 1991

[12] A. Astolfi, R. Ortega, and R. Sepulchre, "Stabilization and disturbance attenuation of nonlinear systems using dissipativity theory," Eur. J. Control, vol. 8, no. 5, pp. 408-431, 2002.

[13] R. Ortega and E. Garcia-Canseco, "Interconnection and damping assignment passivity-based control: A survey," Eur. J. Control, vol. 10, no. 5, pp. 432-450, 2004.

[14] S. L. Shishkin and D. J. Hill, "Dissipativity and global stabilizability of nonlinear systems," in Proc. 34th IEEE Conf. Decis. Control, New Orleans, LA, USA, 1995, pp. 2227-2232.

[15] V. M. Haddad and V. Chellaboina, Nonlinear Dynamical Systems and Control: A Lyapunov-Based Approach. Princeton, NJ, USA: Princeton University Press, 2008.

[16] I. Mizumoto, S. Ohishi, and Z. Iwai, "PFC design realizing output feedback exponential passivity for exponentially stable non-linear systems," IFAC Proceddings Volumes, vol. 40, no. 13, pp. 328-333, 2007.

[17] V. Chellaboina and W. M. Haddad, "Exponentially dissipative nonlinear dynamical systems: A nonlinear extension of strict positive realness," Math. Prob. Eng., vol. 1, pp. 25-45, 2003.

[18] K. Y. Pettersen, "Lyapunov sufficient conditions for uniform semiglobal exponential stability," Automatica, vol. 78, pp. 97-102, Apr. 2017.

[19] S. Xie, L. Xie, and C. E. de Souza, "Robust dissipative control for linear systems with dissipative uncertainty," Int. J. Control, vol. 70, no. 2, pp. 169-191, 1998.

[20] Z. Feng, J. Lam, and Z. Shu, "Dissipative control for linear systems by static output feedback," Int. J. Syst. Sci., vol. 44, no. 8, pp. 1566-1576, 2013.

[21] S. Yuliar and M. R. James, "General dissipative output feedback control for nonlinear systems," in Proc. 34th IEEE Conf. Decis. Control, New Orleans, LA, USA, 1995, pp. 2221-2226.
[22] S. Yuliar, M. R. James, and J. W. Helton, "Dissipative control systems synthesis with full state feedback," Math. Control Signals Systems, vol. 11, pp. 335-356, 1998.

[23] Z.-Y. Feng, J. She, and L. Xu, "A brief review and insights into matrix inequalities for $H_{\infty}$ static-output-feedback control and a local optimal solution," Int. J. Syst. Sci., vol. 50, no. 12, pp. 2292-2305, 2019.

[24] V. L. Syrmos, C. T. Abdallah, P. Dorato, and K. Grigoriadis, "Static output feedback - a survey," Automatica, vol. 33, no. 2, pp. 125-137, Feb. 1997.

[25] V. Veselý, "Static output feedback controller design," Kybernetika, vol. 37, no. 2, pp. 205-221, 2001

[26] C. A. R. Crusius and A. Trofino, "Sufficient LMI conditions for output feedback control problems," IEEE Trans. Autom. Control, vol. 44, no. 5, pp. 1053-1057, May 1999.

[27] Y.-Y. Cao, J. Lam, and Y.-X. Sun, "Static output feedback stabilization: An ILMI approach.” Automatica, vol. 34, no. 12, pp. 1641-1645, Dec. 1998.

[28] M. S. Sadabadi and D. Peaucelle, "From static output feedback to structured robust static output feedback: A survey," Annual Reviews in Control, vol. 42, pp. 11-26, 2016.

[29] H. R. Pota and P. J. Moylan, "Stability of locally dissipative interconnected systems," IEEE Trans. Autom. Control, vol. 38, no. 2, pp. 308312, Feb. 1993

[30] D. de S. Madeira, "Contributions to passivity theory and dissipative control synthesis," Ph.D. dissertation, Dept. Elect. Eng., TU Darmstadt, Darmstadt, Germany, 2018.

[31] R. A. Horn and C. R. Johnson, Matrix Analysis. New York, NY, USA: Cambridge University Press, 2013.

[32] K. R. Stromberg, Introduction to Classical Real Analysis. Belmont, CA USA: Wadsworth, 1981.

[33] S. Boyd, L. El Ghaoui, E. Feron and V. Balakrishnan, Linear Matrix Inequalities in System and Control Theory. Philadelphia, PA, USA: SIAM, 1994.

[34] J. Löfberg, "YALMIP: A toolbox for modeling and optimization in MATLAB," In Proc. IEEE Int. Conf. Robotics Autom., New Orleans, LA, USA, 2004, pp. 284-289.

[35] J. F. Sturm, "Using SeDuMi 1.02, A Matlab toolbox for optimization over symmetric cones," Optimization Methods and Software, vol. 11, no. $1-4$, pp. 625-653, 1999.

[36] X.-M. Yang, "A generalization of a matrix trace inequality," Journal of Mathematical Analysis and Applications, vol. 189, no. 3, pp. 897-900, Feb. 1995.

[37] D. Peaucelle and D. Arzelier, "Ellipsoidal sets for resilient and robust static output feedback," IEEE Trans. Autom. Control, vol. 50, no. 6, pp. 899-904, Jun. 2005.

[38] G. Zhu, J. Levine, and L. Praly, "Stabilization of an electrostatic MEMS including uncontrollable linearization," in Proc. 46th IEEE Conf. Decis. Control, New Orleans, LA, USA, 2007, pp. 2433-2438.

[39] F. Leibfritz and W. Lipinski, "Description of the benchmark examples in $C O M P l_{e} i b$ 1.0," University of Trier, Trier, Germany, Tech. Rep., 2003.

[40] D. de S. Madeira and V. V. Viana, "An application of QSR-dissipativity to the problem of static output feedback robust stabilization of nonlinear systems," in Proc. 23th Congresso Brasileiro de Automática, Porto Alegre, RS, Brazil, vol. 2, no. 1, 2020.

[41] J. W. Simpson-Porco, "Equilibrium-independent dissipativity with quadratic supply rates," IEEE Trans. Autom. Control, vol. 64, no. 4, pp. 1440-1455, Apr. 2019.

[42] P. Apkarian, "Software (hinfstruct, looptune, systune)". Available: http://pierre.apkarian.free.fr/Software.html.

[43] P. Apkarian and D. Noll, "Nonsmooth $H_{\infty}$ Synthesis," IEEE Trans. Autom. Control, vol. 51, no. 1, pp. 71-86, Jan. 2006.

[44] J. V. Burke, D. Henrion, A. S. Lewis, and M. L. Overton, "HIFOO: A MATLAB package for fixed order controller design and $H_{\infty}$ optimization," In Proc. 5th IFAC Symp. Robust Control Design, Tolouse, France, 2006.

[45] G. H. Golub and C. F. Van Loan, Matrix Computations. Baltimore, MD, USA: The Johns Hopkins University Press, 2013.

[46] T. M. Apostol, One-Variable Calculus, with an Introduction to Linear Algebra. New Jersey, NJ, USA: John Wiley and Sons, 1991.

[47] D. de S. Madeira and J. Adamy, "On the equivalence between strict positive realness and strict passivity of linear systems," IEEE Trans. Autom. Control, vol. 61, no. 10, pp. 3091-3095, Oct. 2016. 